\title{
Le Père Paul Le Jeune, s. j., missionnaire-colonisateur (suite et fin)
}

\section{Gilles Chaussé}

Volume 12, numéro 2, septembre 1958

URI : https://id.erudit.org/iderudit/301903ar

DOI : https://doi.org/10.7202/301903ar

Aller au sommaire du numéro

Éditeur(s)

Institut d'histoire de l'Amérique française

ISSN

0035-2357 (imprimé)

1492-1383 (numérique)

Découvrir la revue

Citer cet article

Chaussé, G. (1958). Le Père Paul Le Jeune, s. j., missionnaire-colonisateur (suite et fin). Revue d'histoire de l'Amérique française, 12(2), 217-246.

https://doi.org/10.7202/301903ar d'utilisation que vous pouvez consulter en ligne.

https://apropos.erudit.org/fr/usagers/politique-dutilisation/ 


\title{
LE PĖRE PAUL LE JEUNE, S.J. MISSIONNAIRE-COLONISATEUR *
}

\author{
(suite) \\ Chaptrre VI \\ UN SIĖCLE D'OR
}

«La paix, l'amour \& la bonne intelligence regne parmy nos François. La foy s'estend \& jette de profondes racines parmy les Sauvages, ces quatre paroles suffiroient pour monstrer que nous vivons icy dans un siecle d'or.» $-1^{\text {er }}$ août 1639 : abordent à Québec «un College de Jesuites, une maison d'Hospitalieres, \& un Couvent d'Ursulines 》. - La «participation de la femme à l'évangélisation » des Sauvages: un précédent dans l'histoire missionnaire. - "L'affaire des Hospitalières » se poursuit en France. - La vocation missionnaire de Marie de l'Incarnation. - Un premier fondateur se présente pour le «Seminaire de jeunes filles》. La Réduction de Sillery. - Madame de La Peltrie et les Ursulines. - Le Collège de Québec en 1640. - Le P. Le Jeune et la fondation de Montréal. - La Colonie délègue le $\mathrm{P}$. Le Jeune auprès du Gouvernement français.

Au chapitre second de sa Relation de 1640, intitulé: «De l'estat general de la Colonie françoise, \& de la conversion des Sauvages », le P. Le Jeune résume «l'estat de la Colonie» en ces termes: «La paix, l'amour \& la bonne intelligence regne parmy nos François. La foy s'estend \& jette de profondes racines parmy les Sauvages, ces quatre paroles suffiroient pour monstrer que nous vivons icy dans un siecle d'or. ${ }^{1}$ Il peut paraître à première vue bien téméraire de parler de cette période comme d'un siècle d'or. Cependant, si nous considérons les progrès de la colonie pendant ces dix premières années, avec les yeux d'un homme de la première moitié du XVII ${ }^{\mathrm{e}}$ siècle, si nous comparons, en d'autres termes, le Québec de 1640 non pas avec celui de 1660

* Pour première partie de cet article, voir notre Revue, XII : 56-79.

1 JR, 18: 82 . 
mais avec le Québec de l'arrivée du P. Le Jeune en 1632, il faut reconnaître avec le P. Le Jeune que la Nouvelle-France, en 1640, connaissait, pour ainsi dire, son premier «siècle d'or 》.

«La paix, l'amour \& la bonne intelligence, écrivait le P. Le Jeune, regne parmy nos François. » Nous avons eu, au chapitre précédent, un aperçu de la psychologie qui commandait, au moyen du carcan et du chevalet, la pratique des bonnes mœurs et de la Religion. D'ailleurs, en 1640, Québec ne comptera guère que 150 à 200 habitants ; ${ }^{2}$ les occasions de dispute étaient donc tout à fait limitées. Quant au second aspect: « La foy s'estend \& jette de profondes racines parmy les Sauvages », il n'entre pas, à proprement parler, dans le cadre de cette étude, d'en discuter les progrès. Aussi nous en tiendrons-nous à un autre aspect auquel le $\mathrm{P}$. Le Jeune faisait certainement allusion quand il proclamait «nous vivons icy dans un siecle d'or »: l'essor, étonnant pour l'époque, connu par la colonie au cours des années 16391640. Ce qui n'était en 1636 qu'à l'état de projet, collège, hôpital, couvent, séminaire, était devenu, en 1639-1640, une réalité concrète. "Quand on nous vint donner avis, écrivait le P. Le Jeune en 1639, qu'une barque alloit surgir à Kebec, portant un College de Jesuites, une maison d'Hospitalieres, \& un Couvent d'Ursulines; la premiere nouvelle nous sembla quasi un songe, mais en fin descendans vers le grand fleuve, nous trouvasmes que c'estoit une verité. $\gg^{3}$

Avec la fondation définitive de ces différentes institutions, nous assistons, pour ainsi dire, au couronnement de l'œuvre missionnaire et colonisatrice du P. Le Jeune. ${ }^{4}$ " Point par point,

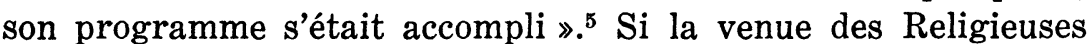
Hospitalières et Ursulines lui semblait «quasi un songe », c'est

2 En 1641, la colonie compte entre 200 et 300 habitants. Si nous soustrayons la population des Trois-Rivières - Salone estime qu'il y avait alors, en 1639, 14 chefs de famille; une cinquantaine de personnes peut-être - nous arrivons pour la ville de Québec au chiffre de 150 à 200 habitants. E. Salone, La Colonisation de la Nouvelle-France, 62-63.

$3 \mathrm{JR}, 16: 18$.

4 Notons qu'au même moment le P. Le Jeune était remplacé dans sa charge de supérieur de toute la Mission canadienne par le P. Barthélemy Vimont. JR, 15: 216.

5 G. Goyau, Les Origines Religieuses du Canada, 148. 
qu'il s'agissait là d'un précédent dans le monde missionnaire à l'époque. «La femme française, en rompant avec une tradition plus que millénaire et qui semblait immuable comme un dogme, prenait alors, écrit Dom Jamet, une initiative sans précédent dans la pratique des nations chrétiennes ${ }^{6}{ }^{6} \mathrm{Si}$ 《ailleurs, la femme avait déjà passé les mers et accompagné l'homme dans le nouveau-monde, elle y était restée ce que l'ancien l'avait connue: la gardienne du foyer, l'être d'intérieur. De France au contraire, continue Dom Jamet, elle venait en missionnaire: pour réclamer le droit à l'accomplissement du devoir que lui imposait son baptême et dont elle avait pris tout à coup la conscience aiguë dans le secret de son cloître. »?

Le plus étonnant dans cette initiative, c'est qu'elle se situait à un moment où la discipline du Concile de Trente tendait à resserrer la clôture autour de la religieuse consacrée et à la remettre "dans l'observance d'une séparation stricte d'avec le monde $\gg{ }^{8}$ D'ailleurs, comment espérer dans ces premières années de la Mission, alors que la colonie se suffisait à peine à elle-même, la présence de la religieuse sur le champ de l'apostolat ? Et, au surplus, où trouver un bienfaiteur pour doter leur couvent ? Que de fois le P. Le Jeune l'avait fait entendre ce cri angoissant: «Faut-il que les biens de la terre, empeschent les biens du ciel $! »^{9}$ On comprend, à l'aide de ce contexte, que le $\mathrm{P}$. Le Jeune se rendant compte, dès son arrivée au pays, qu'une bonne partie de la tâche qui lui était confiée lui échapperait - l'éducation des jeunes filles et les « soins à donner aux malades et aux vieillards » - ait d'abord fait appel à «quelque bonne veusve courageuse» accompagnée de «quelques Compagnes animées de pareil courage $\gg{ }^{10}$ Nous nous expliquons mieux également la surprise éprouvée par le P. Le Jeune à la nouvelle de l'intérêt grandissant

6 Dom Albert Jamet, éd.: Les Annales de l'Hôtel-Dieu de Québec, 1636 1716, composées par les Révérendes Mères Jeanne Françoise Juchereau de S. Ignace et Marie Andrée Duplessis de Ste Hélène, anciennes religieuses de ce monastère (Québec, 1939), III. A l'avenir: Dom Jamet, Les Annales de l'Hôtel-Dieu de Québec.

7 Ibid., III.

8 Ibid., VIII.

9 JR, 7: 224.

10 JR, 5 : 144, JR, 6 : 152. 
suscité à l'intérieur même des cloîtres pour les affaires de la Nouvelle-France. «Ce qui m'étonne, avait-il confié dans sa Relation de 1635, c'est qu'un grand nombre de filles Religieuses, consacrées à nostre Seigneur, veulent estre de la partie; surmontant la crainte naturelle à leur sexe, pour venir secourir les pauvres filles, \& les pauvres femmes des Sauvages. Il y en a tant qui nous écrivent, \& de tant de Monasteres, \& de divers Ordres tresreformez en l'Eglise; que vous diriez que c'est à qui se mocquera la premiere des difficultez de la Mer, des mutineries de l'Ocean, \& de la barbarie de ces contrées $\gg{ }^{11}$ Il y avait donc, à n'en pas douter, une «vocation missionnaire féminine ». ${ }^{12}$

Le plus remarquable, cependant, pour le P. Le Jeune, en 1639, c'est qu'au moment même où « Dieu touchoit à Paris le cœur de madame la Duchesse d'Aiguillon, \& luy inspiroit de bastir un Hostel-Dieu pour les Sauvages qui mouroient dans les bois abandonnez de tout secours, \& qu'elle jettoit les yeux pour ce dessein sur les Religieuses Hospitalieres de Dieppe, il suscitoit en un autre endroit de la France une honneste \& vertueuse Dame, $\&$ l'inspiroit d'entreprendre le Seminaire des petites filles des Sauvages, \& d'en donner le gouvernement aux Ursulines ». $^{13}$

La première de ces deux grandes bienfaitrices, la duchesse d'Aiguillon, nous est déjà bien connue. Nous avons encore présent à l'esprit l'admirable lettre qu'elle faisait parvenir au $P$. Le Jeune, en juin 1636. Dans cette lettre que nous avons qualifiée, au chapitre précédent, d' « une des plus belles pages de la littérature missionnaire qui soit », la duchesse exprimait son désir de fonder prochainement un hôpital en Nouvelle-France et d'y envoyer, à cette fin, «six ouvriers, pour défricher des terres, \& faire quelque logement pour ces bonnes Filles [ les Hospitalières de Dieppe] ».14 Cette fondation, cependant, s'avérait des plus difficiles. «Une grande maison, avait alors répondu Le P. Le Jeune, ne se fait pas bien en peu de temps, \& par un petit nombre de personnes. ${ }^{15}$ D'autant plus que la «pénurie de ressources

$11 \mathrm{JR}, 7: 256$.

12 Dom Jamet, Les Annales de l'Hôtel-Dieu de Québec, IX.

13 JR, $16: 8$.

14 JR, $8: 234$.

$15 \mathrm{JR}, 9: 100$. 
et de bras », à Québec, était encore grande. Aussi entre «l'acceptation enthousiaste» des Hospitalières de Dieppe et leur arrivée en Nouvelle-France, trois années s'étaient écoulées, «trois années d'atermoiements où le $P$. Le Jeune, qui à Québec n'avait toujours pas une logette à offrir, s'employa pour contenir l'héroïque impatience qu'il avait déchaînée à Dieppe et dans le royaume $\gg^{16}$

$\mathrm{Du}$ reste, après un départ prometteur, l'affaire des Hospitalières, fait remarquer Dom Jamet, n'avait fait que peu de progrès en France. ${ }^{17} \mathrm{~A}$ la mi-carême 1636 , - nous l'avons déjà noté au chapitre précédent, - Madame de Combalet, ${ }^{18}$ par l'entremise du P. Chastelain, avait fait appel aux Hospitalières de Dieppe. ${ }^{19}$

Or ce n'est que 18 mois plus tard, soit le 16 août 1637 , qu'elle signait avec le libraire Sébastien Cramoisy, procureur des Hospitalières de Dieppe, le contrat de fondation d'un «Hôtel Dieu dans la Nouvelle France ».20 Quelques mois plus tôt, cependant, le 18 mars 1637, vraisemblablement à la nouvelle de l'acceptation de ces «bonnes Filles», elle avait demandé et obtenu de la compagnie des Cent-Associés deux concessions de terre pour le futur hôpital. ${ }^{21}$ L'une "comprenait sept arpents et demi à prendre dans l'enclos désigné pour faire la future ville de Québec », et l'autre, "soixante arpents, donnés en fief, situés entre le Cap-Rouge et le Coteau Ste-Geneviève ».22 Cette dernière nouvelle était parvenue au $P$. Le Jeune en juin 1637.

Au même moment, le $\mathrm{P}$. Le Jeune voyait arriver à Québec les «six ouvriers» que Madame de Combalet s'était engagée à

16 Dom Jamet, Les Annales de l'Hôtel-Dieu de Québec, XXX.

17 Ibid., XXX.

18 La seigneurie d'Aiguillon ayant été acquise, en 1638, par Madame de Combalet, le roi érigera de nouveau cette seigneurie en duché-pairie et, par brevet du premier janvier 1638, il en fera cadeau à la nièce de Richelieu. Ibid., XII.

19 D'après les Annales des Hospitalières de Dieppe, la duchesse d'Aiguillon, «sur la mi-carême 1636 », aurait confié au "R.P. Chastelain de la Compagnie de Jésus, qui venait à Dieppe pour passer en la NouvelleFrance », «ses lettres et ses intentions 》 pour les Hospitalières de Dieppe. Ibid., 9.
20 Ibid., XVII, 9.
21 Ibid., 10.
22 Ibid., 10. 
faire passer au pays "pour défricher des terres \& faire quelque logement » pour les Hospitalières de Dieppe. « Des ce même tems [1637], écrit l'annaliste de l'Hôtel-Dieu, elle [Madame de Combalet] envoya quelqu'uns de ses gens en Canada, pour en prendre possession. Ils commencerent des l'automne qu'ils arriverent a faire défricher cette terre, qu'ils nommerent Sainte-Marie; et continuant avec beaucoup de charité et d'empressement a travailler pour le futur hôtel Dieu, ils firent découvrir un petit espace de terrain, sur l'emplacement qui y étoit destiné dans la ville $»^{23}$ Le 12 août de l'année suivante, ${ }^{24}$ le P. Le Jeune jugeait les travaux suffisamment avancés pour pouvoir jeter les premiers fondements : "On y travaille maintenant fort \& ferme, écrivait-il dans sa Relation de 1638, selon que le païs le peut permettre, Madame la Duchesse d'Aiguillon qui a jetté les fondemens de ce grand ouvrage, peut dés cette année gouster les fruicts de ses liberalités. ${ }^{25}$ Quant au contrat de fondation signé le 16 août 1637, le P. Le Jeune n'avait pu en prendre connaissance qu'en juin de l'année suivante [1638].

Entre temps, les Hospitalières de Dieppe à qui le P. Le Jeune avait mandé qu'elles lui «envoyassent les noms de celles qui souhaittoient venir en ces contrées, pour commencer cet establissement ${ }^{26}$ s'étaient offertes en si grand nombre qu'il aurait fallu, au témoignage du missionnaire, «marquer les noms de toute la maison $»^{27}$ Désormais, plus rien ne s'opposait à leur venue en Nouvelle-France. Le 2 février 1639, les Hospitalières procédaient à «l'élection de trois dentr'elles » ${ }^{28}$ pour venir fonder l'hôpital à Québec. En avril, le roi confirmait par ses « lettres patentes » le contrat de fondation du 16 août $1637 . .^{29}$ Le $4 \mathrm{mai}$, enfin, les trois religieuses s'embarquaient sur le Saint-Joseph pour aborder, trois mois plus tard, à Québec. ${ }^{30}$ Elles y étaient,

23 Ibid., 10.

24 Ibid., 10-11.

$25 \mathrm{JR}, 14$ : 124-126.

26 JR, 11: 58.

27 Ibid., 60.

28 Dom Jamet, Les Annales de l'Hôtel-Dieu de Québec, 11.

29 Ibid., XXXI.

30 Ibid., XXXI. 
il va sans dire, grandement attendues. "A peine estoient elles descendües du vaisseau, rapporte le P. Le Jeune dans sa Relation de 1640, qu'elles se virent accablées de malades, la sale de l'Hospital estant trop petite, il fallut dresser des cabanes en leur jardin, à la façon des Sauvages ». ${ }^{31}$ Ce rythme se maintint sans interruption jusqu'au mois de mai 1640. Qu'on en juge par les chiffres que nous révèle la Relation de 1640: plus de cent malades hospitalisés; en outre, plus de deux cents autres «soulagez », «soit en passant, soit en y couchant une nuit ou deux ou davantage ». Il s'en était présenté «jusques à dix, douze, vingt, trente à la fois $\gg .^{32}$ Cette réalisation constituait une étape importante dans l'histoire de la colonisation en Nouvelle-France.

Dotée à sa fondation d'une somme de 22,440 livres, ${ }^{33}$ l'HôtelDieu de Québec ne perdra jamais la faveur de l'éminente bienfaitrice. En 1640, la duchesse gratifia de nouveau l'Hôtel-Dieu d'une somme de 18,100 livres. Une année plus tard, elle lui faisait parvenir 1,500 livres. De 1642 à 1653, ses dons oscilleront chaque année, entre 300 et 500 livres. Après 1662 , ceux-ci ne dépasseront guère les 300 livres. Enfin, à la mort de leur bienfaitrice, en 1675, les Hospitalières de Québec seront parmi les 65 légataires à se partager une somme de 122,500 livres. ${ }^{34}$ L'HôtelDieu connaîtra, au cours de cette période, de pénibles moments; les secours envoyés par la duchesse d'Aiguillon se révéleront, dans bien des cas, insuffisants. Néanmoins, l'œuvre du P. Le Jeune aura persévéré contribuant ainsi à l'édification progressive de la colonie.

$31 \mathrm{JR}, 19: 8$. Nous trouvons le même témoignage dans les Annales de l'Hôtel-Dieu. «... des que nous eûmes dressé des lits pour les malades, écrit l'annaliste, on nous en amena un grand nombre, de sorte que la sale se trouvant trop petite, on fit promptement faire un enclos de pieux, ou l'on fit élever quantité de grandes cabanes d'écorces pour y mettre les Sauvages malades. » Dom Jamet, Les Annales de l'Hôtel-Dieu de Québec, 22-23. 32 JR, 19 : 10.

33 Bien que l'annaliste des Hospitalières ne fasse mention que d'une dotation de 20,000 livres, les Archives de l'Hôtel-Dieu de Québec, par contre, indiquent clairement qu'il s'agit d'une dotation de 22,400 livres. A cela, il faut ajouter un revenu de 1,500 livres \& a prendre sur les coches et carosses de Soissons $\gg$. Ce revenu, cependant, était plus apparent que réel. Dom Jamet, Les Annales de l'Hôtel-Dieu de Québec, 9.

34 Ibid., XX-XXI. 
La duchesse d'Aiguillon, on s'en souvient, n'avait pas été seule à compatir au sort de la jeune colonie. En effet, il s'était trouvé, dans le langage savoureux du P. Le Jeune, une «Amazone » pour conduire et établir des Ursulines «en ces derniers confins du monde $\gg^{35}$ Le P. Le Jeune, cependant, aspirait depuis fort longtemps à leur venue en Nouvelle-France. Dès 1633, il faisait appel à la générosité de quelque grande Dame en France "pour fonder un Seminaire de filles, dont la conduitte seroit premierement donnée à quelque bonne veusve courageuse, accompagnée de deux braves filles $\gg^{36}$ On comprend aisément que le $P$. Le Jeune ait attaché tant d'importance à cette idée d'un «Seminaire de filles » dans la colonie. A quoi aurait servi l'éducation donnée aux jeunes Sauvages appelés tôt ou tard à «se marier à des filles ou femmes Sauvages accoustumées à courir dans les bois »? ${ }^{37}$ Les suivre, c'était inévitablement pour eux « retomber dans la barbarie »; les quitter, c'était engendrer «un autre mal fort dangereux $\gg .{ }^{38}$ L'instruction des jeunes filles paraissait donc tout aussi nécessaire que celle des garçons. Même insistance, l'année suivante, dans la Relation de $1634 .{ }^{39}$ En 1635 , c'est un véritable cri d'angoisse que le $\mathrm{P}$. Le Jeune avait lancé en faveur du «Seminaire»; nous avons d'ailleurs, plus d'une fois, fait allusion à cet appel. Personne, cependant à cette date, n'avait encore approché le $\mathrm{P}$. Le Jeune au sujet de cette fondation.

L'année 1636 devait apporter quelques espoirs au P. Le Jeune. Si l'on en croit Dom Jamet, c'est à ce moment qu'il faudrait voir s'établir les premiers contacts entre le $P$. Le Jeune et Marie de l'Incarnation. "Il faut que vous sçachiez, lui écrivait-elle de Tours vraisemblablement en mars ou avril 1636, que la Nouvelle-France commence d'entrer dans les esprits de plusieurs personnes, ce qui me fait croire que Dieu la regarde d'un œil favorable. Helas ! que diriés vous, mon R. Pere, si sa divine Majesté disposoit les affaires en sorte, que nous eussions bien

$35 \mathrm{JR}, 16: 8$.

36 JR, $5: 144$.

37 Ibid.

38 Ibid.

39 JR, $6: 150-152$. 
tost le courage, \& le moyen de vous aller trouver. Je vous diray que si telle est la volonté de Dieu, qu'il n'y a rien en ce monde, qui m'en puisse empescher, quand mesme je devrois estre engloutie des ondes en chemin $\gg .^{40}$ A la suite de cette lettre, le P. Le Jeune n'avait pu que s'exclamer: «Voila le cœur d'une vraye Ursuline, qui me va découvrant les voyes par où son Ordre pourra un jour passer en ces grandes forests. ${ }^{41}$ Nous ne prétendons pas relever ici toutes les phases de la vocation missionnaire de Marie de l'Incarnation. Rappelons-en simplement les principales étapes.

Noël 1633: ${ }^{42}$ Marie de l'Incarnation s'était vue, en songe, marchant au côté d'une dame inconnue et atteignant un «lieu ravissant » d'où lui était apparu un «grand et vaste pays, plein de montagnes, de vallées et de brouillards épais qui remplissaient tout, excepté une petite maisonnette qui était l'église de ce payslà ». Au-dessus de la petite chapelle, se tenait la Vierge, avec, dans ses bras, l'enfant Jésus, regardant « ce pays autant pitoyable qu'effroyable». «Lors, rapporte Marie de l'Incarnation dans ses Ecrits, je la vis devenir flexible et regarder son béni Enfant, auquel sans parler elle faisait entendre quelque chose d'important à mon cœur. Il me semblait qu'elle lui parlait de ce pays et de moi et qu'elle avait quelque dessein à mon sujet. ${ }^{43}$ Plus tard, Marie de l'Incarnation apprendra que ce «grand et vaste pays »

40 Marie de l'Incarnation à Paul Le Jeune, mars-avril 1636, JR, 8: 238. «L'attribution de cette lettre à Marie de l'Incarnation, écrit Dom Jamet, est conjecturale. Cependant, s'empresse-t-il d'ajouter, cette conjecture n'est pas sans quelques probabilités. 》 Dom Albert Jamet, éd.: Marie de l'Incarnation, Ursuline de Tours, fondatrice des Ursulines de la NouvelleFrance. Ecrits spirituels et historiques, publiés par Dom Claude Martin de la Congrégation de St-Maur, réédités par Dom Albert Jamet de la Congrégation de France, avec des annotations critiques, des pièces documentaires et une biographie nouvelle (4 vol., Québec, 1929-1939), 3: 94. A l'avenir: Dom Jamet, Ecrits spirituels et historiques.

41 JR, 8: 238.

42 Dom Jamet qui avait rapporté cette vision dans le Témoignage de Marie de l'Incarnation [Pge. 183] et dans le second tome des Ecrits spirituels et historiques [Pge. 303] aux fêtes de Noël 1634, écrit au tome troisième des Ecrits: «L'étude attentive des lettres de 1635 , contemporaines des faits, ne permet pas de retenir ces conclusions. \ C'est donc bien aux fêtes de Noël de 1633 qu'il faut situer la vision prophétique de Marie de l'Incarnation. Dom Jamet, Ecrits spirituels et historiques, 3: 74.

43 Ibid., 2 : 304-305. 
n'était autre que la Nouvelle-France, et cette compagne inconnue, Madame de La Peltrie. Une année se passe; Marie de l'Incarnation est de nouveau l'objet de la même vision. Cette fois, cependant, la voix de «cette adorable Majesté » est plus explicite: «C'est le Canada que je t'ai fait voir; il faut que tu y ailles faire une maison à Jésus et à Marie. ${ }^{44}$

Or, à la même période, Marie de l'Incarnation recevait du P. Poncet ${ }^{45}$ une Relation de la Nouvelle-France. Cette Relation qui ne pouvait être que celle de 1634 parue, à Paris, au début de l'année 1635, allait orienter de façon définitive la vocation missionnaire de l'Ursuline. Pourquoi ne serait-elle pas cette «brave maistresse» que le P. Le Jeune souhaitait grandement voir passer en Nouvelle-France "pour dresser un seminaire de petites filles » ? ${ }^{46}$ «Plaise à sa divine Majesté, avait alors écrit le $\mathrm{P}$. Le Jeune, d'en inspirer quelques unes, pour une si noble entreprise, \& leur fasse perdre l'apprehension que la foiblesse de leur sexe leur pourroit causer, pour avoir à traverser tant de mers, \& vivre parmy des Barbares. ${ }^{47}$ Marie de l'Incarnation ne pouvait plus hésiter. Cependant, une année entière se passera ${ }^{48}$ avant qu'elle ne fasse part de ses aspirations intérieures au P. Salin, au P. de Lidel, au P. de la Haye, et, par l'entremise de celui-ci, au P. Poncet. ${ }^{49}$ Nous retrouverons ce dernier, plus tard, à un moment capital dans la vie de Marie de l'Incarnation.

Vraisemblablement à l'instigation du P. de la Haye, Marie de l'Incarnation entreprit alors d'écrire au P. Le Jeune, le prin-

44 Ibid., 2 : 316.

45 Ibid., 2: 317 . C'est avant son départ pour Rome, en septembre 1635, que le $\mathrm{P}$. Poncet qui avait été, au collège d'Orléans, le professeur de son fils Claude, lui faisait parvenir cette Relation du P. Le Jeune. Le P. Poncet ne reviendra en France qu'une fois ordonné prêtre, à la fin de l'année 1638. Ibid., 2: 321 .

$46 \mathrm{JR}, 6: 150$.

47 Ibid., 152.

$48 \mathrm{Au}$ cours de cette année, Marie de l'Incarnation se tint en rapport étroit avec un moine Feuillant, Dom Raymond de St-Bernard qui, lui aussi, aspirait fortement passer en Nouvelle-France. Le concours de ce dernier s'étant révélé inefficace, Marie de l'Incarnation se retourna vers les Pères Jésuites. Déjà, cependant, en janvier 1634, elle s'était ouverte à l'un d'eux, le P. Dinet, du songe dont elle avait été l'objet. Dom Jamet, Ecrits spirituels et historiques, $3: 95$.

49 C'est elle-même qui l'écrit: «Il [P. de la Haye] communiqua, comme je l'ai su depuis, mes papiers au Révérend Père Poncet. 》 Ibid., 2: 328. 
cipal intéressé, il va sans dire, dans cette affaire. Nous connaissons sa lettre de 1636 ; celles de 1637 et de 1638 nous sont connues par les réponses ${ }^{50}$ que lui fit parvenir de NouvelleFrance le $P$. Le Jeune. Elles nous révèlent la façon énergique dont le missionnaire usait à l'égard de ses dirigées. ${ }^{51}$ Dans sa réponse $d u$ mois d'août 1637 , le $\mathrm{P}$. Le Jeune lui reprochait sa présomption d'oser aspirer «à des emplois infiniment élevés audessus de ses forces et de son sexe ${ }^{52}$ Même ton en $1638 .{ }^{53}$ «Pour mon regard, écrivait Marie de l'Incarnation à Dom Raymond de St-Bernard, il [P. Le Jeune] ne me parle en aucune manière du Canada, mais il me fait une grande lettre aussi humiliante que la première. N'est-ce pas là un bon Père ? ${ }^{54}$ Le $P$. Le Jeune ne faisait, évidemment, qu'éprouver la vocation de son illustre correspondante. Ainsi pendant que, dans ses lettres, il la mortifiait de la sorte, à Québec, il promettait aux PP. Chastelain et Garnier de faire tout ce qu'il lui serait possible pour donner suite au désir de Marie de l'Incarnation. Ces Pères qui, incidemment, se faisaient les avocats de l'Ursuline auprès du $P$. Le Jeune, le lui avaient appris dans une lettre qu'ils lui adressaient de la résidence de l'Immaculée-Conception en Huronie, au mois d'avril-juin 1637.55 «Quant à ce qui me touche, écrivait-elle la même année à Dom Raymond, le R. P. Paul

50 Marie de l'Incarnation y fait mention dans une lettre à Dom Raymond de St-Bernard. Ibid., 3: 106-109. Dom Jamet a pu, grâce à cette lettre, en reconstituer les grandes lignes.

51 Nous avons à ce sujet mille autres exemples dans ses Lettres spirituelles écrites à plusieurs personnes de piété vivant en religion et dans le monde. F. Fressencourt, éd., Lettres spirituelles écrites à plusieurs personnes de piété vivant en religion et dans le monde touchant la direction de leur intérieur par le R. P. Paul Le Jeune de la Compagnie de Jésus, ancien supérieur de la Mission du Canada (Paris, 1875). Désormais: P. Le Jeune, Lettres spirituelles.

52 Paul Le Jeune à Marie de l'Incarnation, Québec, août-septembre 1637, Dom Jamet, Ecrits spirituels et historiques, 3: 100.

53 Paul Le Jeune à Marie de l'Incarnation, Trois-Rivières, août-septembre 1638, Ibid., 3 : 106.

54 Marie de l'Incarnation à Dom Raymond de St-Bernard, Tours, octobre 1638, Ibid., 3: 106-107.

55 Charles Garnier et Pierre Chastelain à Marie de l'Incarnation, Résidence de l'Immaculée Conception de Notre-Dame, avril-juin 1637, Ibid., 3 : 100. Ces deux Pères, d'après Dom Jamet, auraient été chargés par le P. de la Haye, à leur départ pour la Nouvelle-France, en avril 1636, d'une «commission 》 pour le P. Le Jeune. Ibid., 3: 96. 
Le Jeune a dessein de faire passer des religieuses en ce pays-là pour instruire les petites filles, et ces bons Pères qui m'ont écrit, en ayant entendu parler, l'ont prié de ne me pas laisser. Il leur a promis de faire pour moi tout ce qu'il pourra. Me voilà à présent dans l'espérance et dans l'attente. $\gg^{56}$ Le P. Le Jeune avait-il donc trouvé un bienfaiteur qui doterait enfin la colonie d'un séminaire de filles ? Ecoutons-le nous répondre dans sa Relation de 1637. «Si j'osois violer le secret, je mettrois icy les noms de quantité de personnes, tres relevés en honneur, en vertus, en merites, dont le cœur \& les mains combattent avec nous au ciel, \& en la terre. L'un d'eux voyant qu'on disposoit un Hospital, pour les pauvres Sauvages, jette les fondemens d'un Seminaire de petites filles. $\gg^{57}$

Le 15 janvier 1637, les directeurs de la compagnie des CentAssociés concédaient, en effet, à « Maître Jean de Beauvais, Commissaire de la marine du Ponent, [agissant au nom] d'un personnage de qualité et singulière piété, [dont il avait représenté le dessein] de contribuer à l'établissement et dotation d'une maison de religieuses à Québec, ... l'Ordre desquelles il réservait de nommer dans quelque temps,... qui eussent soin d'élever les jeunes filles des Sauvages et des Français qui résideront en la Nouvelle-France,... une quantité de terres raisonnable pour faire construire l'église et monastère des dites religieuses pour loger les filles des Sauvages et des Français, qui leur pourront être baillées pour les élever ».58 En plus de cette "quantité de terres » «d'une contenance de douze arpents » et qui devait être située dans l'enceinte de Québec, les directeurs de la Compagnie octroyaient au «même sieur de Beauvais » pour les «dites Religieuses à perpétuité, une lieue de terre de longueur sur le Fleuve Saint-Laurent, sur dix lieues de profondeur dans les terres, à prendre au-dessus ou dessous de Québec, en lieu non encore concédé ». Ce personnage anonyme qui avouera lui-même dans une lettre à Monsieur de Montmagny qu'il avait toujours eu «l'intention de n'y être point connu et nommé » nous est précisément

56 Marie de l'Incarnation à Dom Raymond de St-Bernard, Tours, 26 octobre 1637, Ibid., 3 : 102-103.

57 JR, 11: 48 .

58 Dom Jamet, Ecrits spirituels et historiques, $3: 82$. 
connu par cette lettre qu'il adressait au Gouverneur en juin 1637. Dans cette lettre, Noël Brûlart de Sillery témoignait son estime pour la «principale part et conduite » que tenait M. de Montmagny dans les affaires de la Nouvelle-France. C'est pourquoi, ajoutait-il, «je devais confidemment m'ouvrir à vous de l'affection et de l'inspiration que je ressens pour ce même effet ». Puis, il priait instamment le Gouverneur de bien vouloir lui donner «au meilleur endroit qu'il se pourrait dans l'enceinte de Québec » les douze arpents qui lui avaient été octroyés par les directeurs de la compagnie des Cent-Associés et « les autres encore de plus grande étendue aux endroits plus proches de la dite ville », "pour le tout servir et être affecté au bien de la dite mission ». «Le P. Le Jeune, ajoutait-il, me fera cette grâce d'avoir l'œil sur les ouvriers que nous envoyons pour la construction du bâtiment et pour défricher les terres ». Et il terminait sa lettre en ces termes révélateurs: "Obligez moi au passage de la flotte, de me mander sincèrement ce que vous jugez de notre petit dessein en l'établissement de ce séminaire pour instruire et élever en la foi les filles des Sauvages avec les Françaises qui se trouveront dans le pays, et si cela pourra être bien utile et dans quel tems la maison pourra être faite pour servir à ce dessein. \$ $^{59}$

Le P. Le Jeune, cependant, jugeait-il le moment opportun pour procéder à l'établissement d'une telle institution à Québec ? Tout porte à croire qu'il ait songé un instant à la venue immédiate de religieuses enseignantes dans la colonie. Les témoignages des PP. Chastelain et Garnier que nous rapportions tout à l'heure le donneraient à entendre. Cependant, - et c'est ici que s'affirme le réalisme du $\mathrm{P}$. Le Jeune - à quoi aurait, en effet, servi la présence de religieuses enseignantes comme de religieuses hospitalières au milieu d'un peuple non encore sédentarisé ? «Depuis l'embouchure du fleuve S. Laurens jusques à cette Isle [l'Isle de Montreal], écrira le P. Le Jeune plus tard, tous les Sauvages sont errans. ${ }^{60}$ Aussi, concluait-il en 1640 , - et, en

59 F. Martin, éd. et trad., Relation abrégée de quelques Missions des Pères de la Compagnie de Jésus dans la Nouvelle-France par le Père François-Joseph Bressani de la même Compagnie, 296-297. Désormais: Bressani, Relation abrégée.

$60 \mathrm{JR}, 18: 78$. 
1637, il ne pensait pas autrement - «nostre plus grand effort doit tendre à rallier ces pauvres brebis egarées, sans cela il n'y a point d'occupation en ces contrées pour des Religieuses, notamment pour les Ursulines ${ }^{61}$ Quand donc le Chevalier de Sillery écrivait à M. de Montmagny: «jugez si cela pourra être bien utile », il était loin de comprendre les conditions qui régissaient l'activité apostolique en Nouvelle-France. Le P. Le Jeune, avec toute l'adresse dont il était capable, se chargea d'éclairer ce bienfaiteur inattendu.

"Comme nous cherchions toujours, raconte-t-il dans sa Relation de 1638, quelque secours pour faire cette entreprise, [reduire les Sauvages dans une espece de Bourgade] arrive qu'une personne de vertu de vostre France bien cognuë au Ciel \& en la terre, \& dont le nom ne peut sortir de ma plume sans luy deplaire, me donna advis d'un dessein qu'il avoit de servir Nostre Seigneur en ces contrées. ${ }^{62}$ Ce dessein, nous l'avons vu, consistait en la fondation d'un couvent à Québec. Or ce bienfaiteur ayant envoyé " à cét effet quelques artisans \& quelques hommes de travail pour commencer un bastiment, \& pour défricher quelques terres» et ayant assuré le P. Le Jeune dans ses lettres «qu'il n'avoit point d'autre but en ce travail que la plus grande gloire de Dieu ${ }^{63}$ le $\mathrm{P}$. Le Jeune écrivit à ce «bon Seigneur » lui mandant «qu'il feroit un grand sacrifice à Dieu s'il vouloit appliquer le travail de ses hommes à secourir les Sauvages $\gg^{64}$ Entre temps, le P. Le Jeune avait offert à une famille sauvage la maison qu'on était à bâtir à 《une bonne lieuë» au dessus de Kebec》 《à condition neantmoins, prenait-il soin de signifier, à son chef qu'il en sortiroit» si celui à qui il avait écrit «n'en estoit pas content $\gg .65$

La réponse du Chevalier de Sillery parvint au P. Le Jeune en juin 1638. "Cét homme vrayment de Dieu» s'en remettait totalement au P. Le Jeune; il accordait « ce secours aux pauvres Sauvages avec un cœur si denué \& plein d'amour que nous en

61 Ibid.

62 JR, 14 : 204.

63 JR, Ibid., 204.

64 Ibid., 206.

65 Ibid., 206. 
restions, confessait le $\mathrm{P}$. Le Jeune, tous estonnés ${ }^{66}$ C'est en ce sens que le $P$. Le Jeune avait pu, dès 1637, parler d'un bienfaiteur pour la réduction de Sillery ${ }^{67}$ et d'un certain nombre d'ouvriers envoyés à cette fin. Il n'avait fait, en somme, qu'employer pour la réduction les «secours » destinés à l'érection d'un couvent.

L'assentiment donné par M. de Sillery au projet du P. Le Jeune, en juin 1638, n'allait pas s'arrêter là. Jusqu'au mois de février 1639, la résidence de Saint-Joseph de Sillery bénéficiera du même donateur d'une somme de 12,000 livres. L'acte officiel de la fondation, signé à Paris le 22 février 1639 , en témoigne. ${ }^{68}$ De plus, cet Acte accordait à la réduction une autre somme, cette fois, de 20,000 livres, payable à la mort de M. de Sillery. Jusqu'à cette date, l'illustre bienfaiteur s'engageait à verser annuellement à la mission la somme de 1,500 livres. $^{69}$ M. de Sillery, toutefois n'en allait pas moins contribuer à l'établissement d'un «seminaire de jeunes filles » en Nouvelle-France.

Nous avons vu précédemment que M. de Sillery avait obtenu de la compagnie des Cent-Associés deux concessions de terre fort importantes: l'une, «dans l'enceinte de Québec», l'autre, "de plus grande étendue, aux endroits plus proches de la dite ville ». Or la résidence de Saint-Joseph, située à « une bonne lieuë au dessus de Kebec sur le grand fleuve », avait été construite, nous dit la Relation de 1638, en un lieu que «Monsieur Gand avoit pris pour soy » mais qu'il «consacra volontiers à un si bon dessein $\gg^{70}$ Aussi les terres concédées à $M$. de Sillery étant toujours disponibles, les Ursulines en bénéficièrent «pour la construction de leur monastère et de leur séminaire $»{ }^{71} \mathrm{Si}$ le 《 séminaire de jeunes filles » avait perdu en Noël Brûlart de Sillery un

66 Ibid., 214.

67 « Nul doute, écrit le chanoine Groulx, que l'expérience du Paraguay n'ait hanté l'esprit du Père Le Jeune. 》 C'est dans cette expérience, semble$t$-il, que le $P$. Le Jeune aurait puisé son idée d'une réduction en NouvelleFrance. Lionel Groulx, «Missionnaires de l'Est en Nouvelle-France », Revue d'histoire de l'A mérique Française, 3 (1949) : 53.

68 Bressani, Relation abrégée, 298.

69 Ibid., 298-299.

$70 \mathrm{JR}, 14$ : 204-206.

71 Dom Jamet, Ecrits spirituels et historiques, $3: 82$. 
premier bienfaiteur, il ne tarderait pas, cependant, à en trouver un second qui se montrerait, cette fois, plus tenace dans son projet.

«En ce même temps que la divine Majesté m'occupait de la sorte, écrit Marie de l'Incarnation, elle disposait l'esprit de Madame de La Peltrie, personne d'une éminente vertu, pour se donner avec tous ses biens à la Mission de Canada, laquelle avait été puissamment touchée, lisant une Relation en laquelle le Révérend Père Le Jeune, par invitation, disait "s'il ne se trouverait point quelque sainte âme qui voulût aller ramasser le Sang du Fils de Dieu pour le salut des pauvres barbares de ces contrées de Canada. ${ }^{72}$

Ce passage n'était autre que le pressant appel de $1635^{73}$ dont nous avons, plus d'une fois, souligné l'importance jusqu'à maintenant. Veuve à 24 ans, Madame de La Peltrie s'était mise, à la suite de « la lecture des Relations》, à penser «aux moyens de contribuer à l'instruction des petites filles Sauvages ». Elle fit faire à cette intention, nous dit le $P$. Le Jeune, "quantité de prieres ». Pour toute réponse, Dieu «se servit d'une forte maladie» au cours de laquelle Madame de La Peltrie «fit vœu de consacrer ses moyens \& sa personne à la Nouvelle France sans en rien communiquer à personne » si telle était la Volonté Divine. Revenue à la santé, « elle ne fit que penser à l'execution de son dessein.$^{74}$ 《Elle devait hélas! compter avec la tendresse de son père, qui, pour la garder, écrit Goyau, voulait qu'elle se remariât, et compter, aussi, avec les convoitises de toute une famille, peu soucieuse que les héritiers de $M^{\text {me }}$ de La Peltrie fussent plus tard les sauvages. ${ }^{75}$ Madame de La Peltrie, cependant, ne devait pas se troubler pour si peu. "Elle prit resolution de feindre qu'elle vouloit se remarier, \& par ce moyen se remit en la bonne grace de son Pere. ${ }^{76}$

Ayant donc gagné Paris en compagnie de M. de Bernières dans le but de réaliser son « pieux dessein », Madame de La Peltrie

72 Ibid., 2: 318.

73 JR, $7:$ 258-260.

74 JR, 16: 10-12.

75 G. Goyau, Les Origines religieuses du Canada, 140.

76 JR, 16: 12. 
" conféra de son entreprise avec plusieurs saints \& doctes personnages qui l'approuverent tous $\gg{ }^{77}$ Parmi ces $«$ saints \& doctes personnages » il nous faut mentionner, outre le $\mathrm{P}$. de Condren, supérieur de l'Oratoire et M. Vincent, supérieur de Saint-Lazare, le P. Poncet, depuis peu le confident de Marie de l'Incarnation, ${ }^{78}$ le P. Dinet et le P. de la Haye. "Ce dernier, écrit Marie de l'Incarnation dans sa Relation de 1654, chargea le Révérend Père Poncet de m'écrire tout ce qui se passait, car madite Dame [Madame de La Peltrie] et moi-même nous ne nous connaissions point encore ni de réputation, ni autrement, que ce que les dits Révérends Pères lui avaient dit à mon insu $\gg^{79}$ Le 15 novembre 1638, le P. Poncet écrivait à Marie de l'Incarnation et lui faisait part «des intentions de cette Dame pour la fondation d'une maison de religieuses et d'un séminaire de filles sauvages à Québec ». «Il croyait, ajoutait-il, que le temps était venu auquel Dieu (se proposait) d'accomplir son dessein sur elle $\gg .80$ En janvier 1639, Madame de La Peltrie annonçait à l'Ursuline de Tours « l'heureux succès de ses négociations à Paris avec les Pères Jésuites et les Directeurs de la Compagnie des Cent-Associés touchant l'affaire de sa future fondation à Québec $\gg .{ }^{81}$ Plus rien, désormais, ne faisait obstacle à son départ pour la Nouvelle-France. Ce départ devait avoir lieu le 4 mai suivant. ${ }^{82}$

Dès leur arrivée à Québec, Marie de l'Incarnation et ses deux compagnes reçurent dans leur «petit Seminaire » leurs premières recrues: "six filles sauvages ». De plus, «quelques filles Françoises commencerent de les aller voir pour estre instruittes ».83 Bientôt, cependant, « au lieu de six » pensionnaires, c'était dix-huit qu'on pouvait compter «dans cette petite mai-

77 Ibid.

78 Dom Jamet, Ecrits spirituels et historiques, 2: 328. C'est M. de Bernières qui rentrera, le premier, en relation avec le P. Poncet. Ibid., 2: 339-340.

79 Ibid., $2: 340$.

80 Antoine-Joseph Poncet à Marie de l'Incarnation, Paris, 15 novembre 1638, Ibid., 3: 109.

81 Madame de La Peltrie à Marie de l'Incarnation, Paris, 15-17 janvier 1639, Ibid., 3: 117.

82 Ibid., 2: 352.

83 JR, 16: 22. 
son ». «Il est vray, précise le P. Le Jeune, qu'elles ne demeurèrent pas «toutes ensemble à mesme temps, mais pour l'ordinaire, elles estoient six ou sept logées avec Madame de la Pelletrie, trois Religieuses, \& deux filles Françoises, \& tout cela dans deux petites chambres $\gg{ }^{84}$ Ce progrès qui s'était réalisé en une seule année seulement permettait d'augurer déjà ce que serait l'œuvre de demain.

Au côté du «Seminaire des Ursulines » se dressait, on s'en souvient, le collège de Québec. Nous avons longuement raconté aux chapitres précédents les circonstances qui entourèrent la fondation de cette institution. Dès 1635, avions-nous écrit, les PP. Charles Lalemant et Jean de Quen inauguraient les premières leçons publiques au collège. Or voici qu'en 1639 , le $P$. Le Jeune annonçait dans sa Relation «qu'une barque portant un College de Jesuites alloit surgir à Kebec». De quels effectifs pouvait-il bien s'agir ? Rappelons d'abord ce qui s'était passé depuis 1636. Cette année-là, celle où le $P$. Le Jeune apprenait du T. R. P. Général que des «lettres patentes » de Fondateur avait été expédiées au Marquis de Gamache, il écrivait au P. Vitelleschi: "Quant au collège qui a été rendu possible grâce à la libéralité du Marquis de Gamache, nous y instituerons, aussitôt qu'il sera avancé, deux classes: l'une pour les petits Français, l'autre pour les petits Sauvages ${ }^{85}$ Même idée dans la Relation de 1636; le P. Le Jeune apportait, cependant, une nuance. Ce n'était plus deux classes mais trois qu'il envisageait d'instituer dans le nouveau collège lorsqu'il serait construit. «J'espere, si nous pouvons avoir du logement, de voir trois classes à Kébec: la premiere de petits François, qui seront peut-estre vingt ou trente Escoliers: la seconde de quelques Hurons: la troisiéme de Montagnés ${ }^{86}$ L'année suivante, on croirait presque réalisés les désirs du $P$. Le Jeune. "J'oubliois quasi de dire, écrivait-il dans la Relation de 1637, que nous avons parlé de Dieu en sa

84 JR, $19: 36$.

85 Paul Le Jeune au T. R. P. Mutius Vitelleschi, Québec, 8 août 1636. ACSM, 749: 11.

86 JR, 9: 106. 
maison, en langue Latine, Françoise, Montagnése, \& Huronne. $~^{87}$ Toutefois, il ne s'agissait encore que de simples classes de catéchisme. ${ }^{88}$ En 1640, toutefois, Marie de l'Incarnation fera allusion, dans une de ses lettres, "à ces jeunes enfants qui vont au collège pour apprendre le latin $\gg .^{89}$ C'est donc qu'on $\mathrm{y}$ enseignait les premières classes de Lettres. Que le nombre d'élèves ait été relativement peu élevé - en 1636, le P. Le Jeune espérait en avoir de 20 à $30-$, il n'en fallait pas moins un personnel enseignant. Aussi des six Jésuites qui abordaient en août 1639 à Québec, - les PP. Vimont, Chaumonot, Poncet, Gondoin, Bargon et le F. Claude Jager - il faut croire que quelques-uns étaient destinés au Collège. ${ }^{90}$ On pourra objecter, à la lumière de ces faits, que le collège en était encore, en 1640, à ses débuts. Peut-être: seulement les débuts de 1640 contrastaient singulièrement avec ceux de 1635. Il s'était donc accompli, pendant ces cinq premières années, un réel progrès.

Il nous resterait, pour être plus complet, à étudier l'influence des Relations du P. Le Jeune sur la fondation de Montréal. Nous le ferons brièvement. Pour Salone, notons-le immédiatement, il semble n'exister aucun doute quant à l'influence prépondérante que les Relations du P. Le Jeune auraient joué sur la fondation de Montréal. Lorsqu'il s'agit de déterminer le nombre de "véritables colons» que la réclame du $\mathrm{P}$. Le Jeune aurait «pu procurer au Canada», Salone admet que «le problème est difficile à résoudre ». "Mais ce qu'il y a de certain, s'empresse-t-il d'ajouter, c'est que la fondation de Montréal est due à la propagande des Jésuites. ${ }^{91}$ Remarquons que l'historien de la Colonisation de la Nouvelle-France ne nie pas pour autant

$87 \mathrm{JR}, 11: 72$.

88 La même année, le $P$. Le Jeune écrivait au T. R. P. Général: \& On apprend aux enfants le Symbole des apôtres et les autres prières essentielles en français, en latin, en montagnais et en huron. $>$ Paul Le Jeune au T. R. P. Vitelleschi, Trois-Rivières, 23 août 1637, ACSM, 749: 15.

89 Marie de l'Incarnation à la Mère Marie-Gillette Roland, religieuse de la Visitation de Tours, Québec, 4 septembre 1640, Dom Jamet, Ecrits spirituels et historiques, $3: 191$.

90 Voir à ce sujet: Dom Jamet, Les Annales de l'Hôtel-Dieu de Québec, 15,18 , et A. Melançon, Liste des missionnaires Jésuites, Nouvelle-Fraxce et Louisiane (Montréal, 1929), 76.

91 E. Salone, La Colonisation de la Nouvelle-France, 73. 
que $M$. de La Dauversière ait été le «véritable fondateur de Montréal ${ }^{92}$ Seulement le cadre dans lequel s'est déroulée la vie de Jérôme Le Royer de La Dauversière est, pour lui, trop bien ordonné pour qu'on ne puisse y voir aucune influence extérieure. Ainsi fait remarquer Salone, le jeune de La Dauversière fera ses études au collège de La Flèche, le plus célèbre alors des collèges des Jésuites. Pure coïncidence, de plus, si parmi les condisciples $\mathrm{du}$ futur fondateur de Montréal on retrouve un Charles Lalemant, un Anne de Nouë, un Barthélemy Vimont, un Paul Le Jeune ? ${ }^{93}$ Quant aux Relations du P. Le Jeune, sans vouloir leur attribuer un rôle qu'elles n'auraient pas joué, il faut bien reconnaître qu'elles auront grandement aidé le fondateur de Montréal « à trouver sa voie et à préciser la forme de son apostolat 》 ${ }^{94}$ en terre canadienne. Qu'on relise, pour s'en convaincre, le chapitre III de la Relation de 1635 intitulé: "Que c'est un bien pour l'une \& l'autre France, d'envoyer ici des colonies » et où le P. Le Jeune « insinuait, en somme, la fondation d'organismes nouveaux » qui assureraient le peuplement de la colonie, et, par là, la conversion des Sauvages. ${ }^{95}$ Que dire également de tous ces textes relatifs à la fondation d'un hôpital en Nouvelle-France, textes que nous avons d'ailleurs abondamment cités dans ce travail. "Appelé à fonder un hôpital dans la Nouvelle-France », comment $M$. de La Dauversière ne les aurait-il pas lus « avec une sainte émotion $\gg .{ }^{96}$ Et cet autre texte capital - tiré de la Relation de 1636 - où le P. Le Jeune s'était attardé, comme volontairement, à décrire cette "grande Isle nommée de MontReal » et ses environs. ${ }^{97}$ 《Quelle consolation » enfin pour M. de La Dauversière que «l'autorité et l'expérience» avec laquelle s'était prononcé le P. Le Jeune, dans ses Relations de 1640 et de

92 Ibid., 73.

93 Ibid., 75, C. de Rochemonteix, Les Jésuites et la Nouvelle-France au XVIIe siècle, 1: 152-158, 170-190, et L. Pouliot, Etude sur les Relations des Jésuites, 124.

94 Ibid., 125.

95 JR, 8: 8, et L. Pouliot, Etude sur les Relations des Jésuites, 126. 96 Ibid.

97 JR, 12: 132. Ce texte faisait suite au récit du voyage qu'avait fait le $P$. Le Jeune en compagnie du Gouverneur, M. de Montmagny, jusqu'à l'île de Montréal. 
1641, « en faveur du projet de Montréal »!98 Et nous n'avons cité que les textes les plus importants. Le P. Le Jeune n'avait donc pas été étranger, loin de là, à la fondation de Montréal.

Enfin, c'est encore au P. Le Jeune qu'on aura recours, d'abord en 1641, puis de nouveau en 1643, pour plaider, devant les dirigeants de l'Etat français, la cause et les intérêts du Canada. Cette double mission en France met, pour ainsi dire, un premier terme à la vocation colonisatrice du P. Le Jeune. Nous verrons plus loin qu'il faudra considérer, dans la vie du $P$. Le Jeune, une seconde étape où il se révélera de nouveau un ardent colonisateur. A Paris, le P. Le Jeune, il va sans dire, approcha son illustre bienfaitrice, la duchesse d'Aiguillon. Celle-ci, à son tour, intercéda auprès de son oncle, le cardinal de Richelieu. Aussi, quand le $\mathrm{P}$. Le Jeune revint au printemps de 1642, c'est de bonnes nouvelles qu'il apportait de la mère-patrie.

La crainte qu'on avoit des Hiroquois, lisons-nous dans la relation de 1642, avoit tellement abbatu les cœurs, qu'on ne vivoit que dans les apprehensions de la mort; mais si tost que la nouvelle fut venuë qu'on alloit dresser des fortifications sur les avenuës des Hiroquois, toute crainte cessa, chacun reprit courage, \& commença à marcher teste levé, avec autant d'asseurance que si le Fort eust desja esté basty. ${ }^{99}$

Richelieu dépêcha aussitôt dans la colonie 30 à 40 hommes "pour garder le fort qui portait son nom ». En même temps, «il faisait remettre aux Jésuites, par l'entremise de la duchesse d'Aiguillon, des secours pour leurs missions ».100 Le P. Le Jeune, dans une lettre au T. R. P. Vitelleschi, nous apprend luimême en quoi consistaient ces secours. «Le Cardinal de Richelieu, écrivait-il de Dieppe, en avril 1642, à la demande de sa nièce et de $M$. desnoiers a accordé $30,000 £$ du trésor pour construire chez les Hurons un fort très puissant. ${ }^{101}$ Le P. Le Jeune avait donc réussi dans sa mission. En 1643, nous l'avons dit

98 JR, 18: 246-248, JR, 21: 106-108, et L. Pouliot, Etude sur les Relations des Jésuites, 129.

99 JR, 22: 32-34.

100 L. Gérin, Aux Sources de notre Histoire, 140.

101 Paul Le Jeune au T. R. P. Mutius Vitelleschi, Dieppe, 2-23 avril 1642, ACSM, $149: 7$ et $749: 26$. 
précédemment, le $\mathrm{P}$. Le Jeune devait retourner de nouveau en France "pour la même raison». "Les Hiroquois, écrivait-il au T. R. P. Vitelleschi en novembre 1643 , deviennent de plus en plus terribles... Ils veulent même exterminer les français. $\gg 102$ Quels furent les résultats de cette seconde mission ? En 1644, la reine Anne d'Autriche - inspirée, il est vrai, en grande partie par le baron de Renty - consentait à Pierre Le Gardeur de Repentigny un prêt de 30,000 livres « pour employer à la levée des gens de guerre destinés par Sa Majesté pour s'opposer aux incursions des Iroquois ». ${ }^{103}$ De plus, la Reine envoyait une compagnie de soixante soldats sous le commandement de La Barre avec 100,000 livres pour les équiper. ${ }^{104}$ Le P. Le Jeune avait, de nouveau, réussi dans sa mission.

\section{CONCLUSION}

Après nous être longuement penché sur l'état de la colonie au moment où le $P$. Le Jeune exerce ses fonctions de supérieur de la Mission canadienne, il nous faut maintenant conclure. Une première déduction s'impose: nous aurons, sans aucun doute, "remarqué les merveilleuses qualités du P. Le Jeune comme missionnaire-colonisateur ». ${ }^{1}$ Ces "merveilleuses qualités», le $P$. Le Jeune les a tour à tour déployées devant nous, en intercédant auprès du cardinal de Richelieu en faveur de la compagnie des Cent-Associés, en entreprenant lui-même de persuader les colons français d'émigrer en Nouvelle-France, en contribuant, enfin, à faciliter et à hâter leur passage par l'établissement dans la colonie d'institutions sociales indispensables à toute vie communautaire. Autant d'efforts colonisateurs qui, à notre sens,

102 Paul Le Jeune au T. R. P. Mutius Vitelleschi, Larochelle, 25 novembre 1643, ACSM, 749: 32 .

103 Jean Bruchesi, «Le sort des Armes 》, Cahiers de l'Académie Canadienne-Française, 2 (1957) : 174.

104 L. Gérin, Aux Sources de notre Histoire, 140 et Claude de Bonnault, - La Compagnie du Saint-Sacrement - le baron de Renty et le Canada», Bulletin des Recherches Historiques, 38 (1932) : 342.

1 Nous rejoignons, ici, la conclusion d'un grand spécialiste des Relations et des premières années de la colonie, le P. Léon Pouliot. Etude sur les Relations des Jésuites, 261. 
font du $\mathrm{P}$. Le Jeune une des figures dominantes sur la scène de la Nouvelle-France dans la période 1633-1643.

Pour plusieurs, il pourra paraître audacieux de parler des réalisations du $\mathrm{P}$. Le Jeune comme autant d'efforts intimement liés à l'idée de colonisation au pays. On prêtera volontiers au $P$. Le Jeune un rôle de colonisateur quand, par exemple, dans ses Relations de 1635 et de 1636, il fait appel directement aux colons français; ou encore lorsqu'il les reçoit lui-même en terre canadienne. Mais on comprendra mal qu'on puisse se permettre de qualifier d'efforts colonisateurs les autres activités du missionnaire qui, apparemment, n'ont aucune relation avec la culture du sol. Toute l'idée de ce que nous devons entendre par une véritable colonisation est ici engagée.

Cette idée qui donne lieu trop souvent à de fausses conceptions, a été développée de façon très heureuse dans une étude sur la colonisation du Canada au XVIII ${ }^{e}$ siècle. «Il ne faut pas confondre, fait très justement remarquer $M$. Frégault dans cette étude, colonisation et mise en culture du sol. L'expansion agricole reste assurément un aspect important de la colonisation économique. Celle-ci, toutefois, comporte bien d'autres aspects. » «La France, continue M. Frégault, colonise le Canada lorsqu'elle $\mathbf{y}$ envoie des hommes et des femmes qui deviennent canadiens; qu'elle y expédie aussi des chefs politiques, des religieux, des éducateurs, des troupes et, avec ces hommes, des idées, des techniques, des capitaux, c'est toujours une colonisation qu'elle opère. $»^{2}$ Ainsi quand le $P$. Le Jeune écrivait, en 1635, au cardinal de Richelieu pour l'inciter à prêter son appui à la compagnie des Cent-Associés, quand il faisait appel aux religieuses hospitalières et enseignantes pour établir dans la colonie un hôpital et un couvent pour jeunes filles, et qu'il procédait à l'établissement de ces différentes institutions en même temps qu'à celui d'un collège, quand, enfin, il s'embarquait pour la France, d'abord en 1641 , puis de nouveau en 1643 , pour plaider la cause de la colonie devant les principaux dirigeants de l'Etat, le P. Le

2 Guy Frégault, \&a Colonisation du Canada au XVIII siècle s, Cahiers de l'Académie Canadienne-Française, 2 (1957) : 55-56. 
Jeune se révélait un colonisateur beaucoup plus actif et beaucoup plus important que le simple paysan occupé uniquement à son petit coin de terre en Nouvelle-France. Dans cette perspective, l'œuvre colonisatrice du P. Le Jeune prend une singulière grandeur. Aussi, le véritable problème nous paraît plutôt se poser quant à la portée de l'œuvre missionnaire et colonisatrice du $P$. Le Jeune dans la période 1633-1643.

Or si nous en croyons une étude magistrale sur ce sujet, La Colonisation de la Nouvelle-France d'Émile Salone, la période 1633-1643 constituerait une période décisive dans l'histoire coloniale de la Nouvelle-France. "En somme, écrit Salone, à la condition de ne point considérer les résultats en eux-mêmes et de s'en tenir à comparer ce qu'était le Canada en 1633 et ce qu'il est en 1643, un progrès considérable apparaît. A vrai dire, c'est dans ces dix années que la colonie est née. ${ }^{3}$ En 1643 , - les chapitres précédents en témoignent - sont définitivement fondées, ce que nous avons appelé avec Louis-Jaray, « les grandes œuvres des villes », école, collège, couvent, hôpital. De plus, là où le missionnaire n'avait trouvé, à son arrivée, qu'une seule famille et qui se préparait, d'ailleurs, à repasser en France, on compte, en 1643 , entre 200 et 300 habitants «solidement établis ».4 Enfin, « les trois habitations fortifiées - Québec, TroisRivières et Montréal - entre lesquelles » se répartissait ce noyau de population donnait réellement à la France, en même temps

3 E. Salone, La Colonisation de la Nouvelle-France, 82.

4 En 1641, nous avons, d'après Dollier de Casson, 240 habitants en Nouvelle-France; \& ce qui, ajoute Salone, en défalquant le premier convoi des Montréalistes débarqué cette même année, réduit tout juste à deux cents pour 1640 les habitants de la Nouvelle-France ». E. Salone, La Colonisation de la Nouvelle-France, 63. Ferland accepte cette évaluation: sur les 417 colons [chefs de famille] venus en Nouvelle-France dans la période 16151666 , il en compte 85 pour la période précédent 1641 , et 332 pour celle de 1642-1666. J.-B.-A. Ferland, Cours d'histoire du Canada, 1: 510-515. Rameau la trouve cependant trop faible; * il n'admet pas, écrit Salone, que les cinquante-deux chefs de famille dont il constate la présence dans la colonie dans cette période (avant 1641), représentent avec leurs femmes et leurs enfants une population totale de moins de trois cents personnes. E. Salone, La Colonisation de la Nouvelle-France, 63. Cette dernière assertion est confirmée par l'abbé Stanislas A. Lortie qui, dans son étude sur "l'origine des Canadiens-Français », arrive au chiffre de 296 habitants pour la période 1608-1640. «De l'origine des Canadiens-Français 》, Bulletin des Recherches Historiques [RRH] 2 (1903): 18. 
que «la maîtrise du bassin de Saint-Laurent,$^{5}$ une première teinte de maturité.

Si modestes soient-ils, ces humbles débuts prennent, néanmoins, une importance capitale quand on considère, un instant, la terrible menace qui planait alors sur la colonie. Toujours à redouter dans les premières années de la colonie, la menace iroquoise devait atteindre, à partir de 1643, son sommet. «Les Iroquois, que l'expérience rendait tous les jours plus habiles dans la guerre de surprises, avaient, en effet, écrit Rochemonteix, changé de tactique, et divisé leurs forces, dès 1643, en dix bandes de guerriers, destinées à envelopper toute la Colonie française comme dans un immense réseau. ${ }^{6}$ L'arrêt de mort de la colonie était signé. "Pendant plus de vingt ans, écrit le P. Pouliot, les Relations décriront, dans leurs plus horribles détails, les cruautés des Iroquois; pendant plus de vingt ans, elles feront entendre sur tous les tons qu'ils sont le grand obstacle au progrès de la foi et au développement de la Colonie. ${ }^{7}$ Toutefois, - et il y a lieu ici de nous étonner quand on considère le peu de secours qui viendra de France tout au long de cette période, - la petite colonie aura, pendant ces vingt ans, résisté à des ennemis aussi déroutants que cruels qui « venaient en renards, attaquaient en lions et fuyaient en oiseaux 》, agissant « le plus souvent à coup

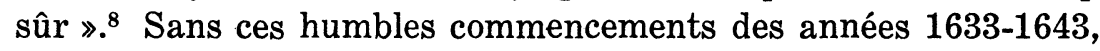
il est à se demander si la petite colonie aurait pu opposer à la menace iroquoise la même force et le même courage intrépide. Façonnée au cours de ces dix premières années, la petite colonie devait prouver dans cette guerre menée sans merci la véritable valeur de ses fondations.

Précisons quelques-unes de ces valeurs. En 1645, avonsnous dit plus haut, étaient définitivement fondés l'école, le collège, le couvent et l'hôpital. On ne saurait trop insister sur l'importance de telles institutions dans une société en formation.

5 Ibid., 82.

${ }^{6}$ C. de Rochemonteix, Les Jésuites et la Nouvelle-France au XVII siècle, 2: 34 .

7 L. Pouliot, Etude sur les Relations des Jésuites, 261.

8 P.F.X. de Charlevoix, Histoire et description générale de la NouvelleFrance, 3: 202. 
Une société - nous avons eu l'occasion de le signaler au cours de ce travail - comporte nécessairement avec elle toute une série d'institutions, politique, sociale, civile, religieuse, etc..., et dans la mesure où elle réalise le plus possible son unité, où elle procure, en somme, à son organisme ces besoins essentiels, elle fait un pas vers sa future maturité. Enrichie, depuis 1639, de ces différentes institutions, la société canadienne se constituait peu à peu.

De plus, parmi les 200 à 300 colons que comptait la Nouvelle-France, on pouvait commencer à distinguer une classe dirigeante. Autre trait qui révèle bien le dynamisme d'une société. On conçoit que les premiers immigrants au pays aient rencontré certaines difficultés; difficultés provenant en partie d'eux-mêmes - leur formation souvent ne les avait pas préparés à ce genre de travail - et en partie, également, d'un sol qui ne se montrait pas toujours clément. Aussi, note fort à propos Gérin, il ne faut pas se surprendre de voir «les colons de premier arrivage se signaler, surtout, par leur main-mise sur les emplois publics » quoique « rares et de faible rapport dans ces commencements $» .^{9}$

Etudions, à titre d'exemple, le cas des premiers arrivants de 1636: les Legardeur et les Leneuf. Les premiers se fixeront à Québec; les seconds, aux Trois-Rivières. " C'est-à-dire, fait remarquer Gérin, qu'ils se partagèrent les seuls postes établis jusqu'alors ».10 Bientôt, ces deux familles s'illustreront aux plus hautes charges de la colonie. Ainsi, Pierre de Repentigny deviendra amiral de la flotte et Michel Leneuf du Hérisson, gouverneur, en 1648, des Trois-Rivières. ${ }^{11}$ En 1645, nous les verrons se substituer à la compagnie des Cent-Associés pour exercer seuls le monopole de la traite sur le Saint-Laurent. La première compagnie d'origine canadienne au pays était née. Rien d'étonnant que nous retrouvions à la tête de cette compagnie les Tilly et les Leneuf. ${ }^{12}$ D'ailleurs, la mission dépêchée à Paris par les Habitants à l'automne de 1644 comptait, il va sans dire, un Repen-

${ }^{9}$ L. Gérin, Aux Sources de notre Histoire, 135.

10 Ibid., 136.

11 Ibid., 150.

12 H. Gosselin, « La Compagnie des Habitants », BRH, 8 (1902) : 279. 
tigny, Pierre de Repentigny, qui s'était fait gloire de se présenter devant les autorités françaises comme «député des habitants du Canada ».13 Cette dernière remarque, pour peu que nous y prêtions attention, est très significative: elle révèle l'existence d'une pensée authentiquement canadienne. La compagnie des Habitants marquait au pays le triomphe de la "gentilhommerie fonctionnariste». Nous tenions à le souligner: la petite colonie de 1643 comptait déjà assez d'éléments dynamiques pour s'affirmer comme telle. Cette affirmation, plus encore que l'importance des résultats qui s'ensuivront, nous incitent fortement à conclure que la première phase, la plus difficile peut-être à franchir pour une colonie qui entend s'organiser sur place, était désormais accomplie. Aussi, cette première période 16331643 nous paraît-elle réaliser, grâce à ses cadres politiques et sociaux déjà bien définis, une unité globale. En somme, les fondements de la colonie étaient posés; il ne restait plus qu'à les consolider par une immigration continue. Pour y parvenir, c'est le P. Pouliot qui le note, «il suffira de rappeler chaque année, sans y insister autant, les avantages temporels et spirituels qu'offre le Canada $\gg .^{14}$

Ce bref aperçu de la portée colonisatrice de l'œuvre du P. Le Jeune dans la période 1633-1643 suffirait à démontrer le rôle de premier plan joué par le missionnaire dans la naissance de la colonie canadienne. Nous pourrions nous arrêter là; notre étude, d'ailleurs, ne prétend aucunement dépasser ce cadre restreint de la vie du P. Le Jeune. Néanmoins, nous nous permettrons de signaler dans la vie du $P$. Le Jeune une autre étape où son activité, cette fois comme procureur de la Mission canadienne, ${ }^{15}$ se fera de nouveau considérablement sentir sur la destinée de la colonie.

13 Claude de Bonnault, \& La Compagnie du Saint-Sacrement - le baron de Renty et le Canada 》, BRH, 38 (1932): 343.

14 L. Pouliot, Etude sur les Relations des Jésuites, 261.

15 \& En 1649, écrit le P. Fressencourt, le P. Le Jeune quitta définitivement la Nouvelle-France; il était appelé à Paris pour gérer la procure de la mission. Les rapports qu'il avait entretenus, ses connaissances particulières, son habileté dans le maniement des affaires le rendaient admirablement propre à cet emploi. 》 Paul Le Jeune, Lettres spirituelles, XX. 
Certains historiens ont prétendu que les Relations, après 1642 , avaient plus fait pour le peuplement de la colonie que les Cent-Associés. «A partir de la fondation de Montréal, écrit en effet Langlois, la propagande des Relations des Jésuites aida plus au peuplement que la compagnie des Cent-Associés, qui sembla s'en désintéresser totalement. ${ }^{16}$ Examinons de plus près cette affirmation. Que les Cent-Associés se soient désintéressés de la Nouvelle-France, il n'y a là rien d'étonnant. Cette compagnie - l'état des finances reproduit à la fin du chapitre troisième en témoigne - était tout simplement ruinée.

D'ailleurs elle avait, depuis 1645 , cédé à la compagnie des Habitants en même temps que «le droit de faire la traite, le soin d'entretenir, de défendre et même de coloniser la colonie ».17 Que la propagande des Relations des Jésuites ait aidé, cependant, plus au peuplement que la compagnie des Cent-Associés, voilà qui pourrait offrir quelque difficulté. Essayons de nous figurer un peu, suggère Salone en faisant allusion aux horreurs de la guerre avec les Iroquois, "l'état d'esprit des lecteurs des Relations qui assistent à de si effroyables spectacles. Pour s'embarquer sur les vaisseaux de la compagnie, ajoute-t-il, il n'y aura bientôt que les religieux qui aspirent au martyre ${ }^{18}$ Tellement que Benjamin Sulte rendra «les Relations en grande partie responsable de l'arrêt du mouvement colonisateur pendant la menace iroquoise $\gg{ }^{19}$ Pourtant, si l'on en juge par les conclusions auxquelles arrive Salone, il est indiscutable que « de 1640 à 1663, loin d'avoir été interrompue, l'immigration a puissamment contribué au peuplement de la colonie ${ }^{20}$ A qui donc attribuer l'initiative de ce mouvement d'immigration ? A la compagnie des Cent-Associés ? Nous avons déjà vu ce qu'il fallait en penser. A la compagnie des Habitants? Pas davantage. Les problèmes financiers auxquels les membres de cette compagnie avaient à faire face ne leur permettaient guère de s'acquitter de cette dernière obligation. $\mathrm{Au}$ Roi ? La suite de ce travail nous aura vite convaincu du

16 G. Langlois, Histoire de la population canadienne-française, 41. 17 E. Salone, La Colonisation de la Nouvelle-France, 100, 104, 127. 18 Ibid., 99.

19 L. Pouliot, Etude sur les Relations des Jésuites, 266, 313.

20 E. Salone, La Colonisation de la Nouvelle-France, 111. 
contraire. Aux Relations alors ? Quoi qu'il en soit, il se trouve que dans la période qui vaut vraiment la peine d'être étudiée au point de vue peuplement après 1642 c'est-à-dire la période 1654$1660,{ }^{21}$ nous voyons de nouveau le $P$. Le Jeune prendre une part active à la rédaction des Relations, particulièrement à celles de 1651-1652, 1652-1653, 1654-1655, 1656-1657 et 1657-1658. ${ }^{22}$

Enfin, si comme le prétend Salone, les Jésuites ont puissamment contribué, grâce à leurs appels incessants, à sauver la Nouvelle-France de la menace iroquoise, ${ }^{23}$ le plus pressant de ces appels est, sans contredit, « l'épitre que le père Le Jeune adressait

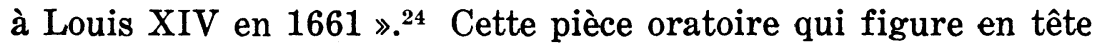
de la Relation de 1661, est trop belle pour que nous n'en reproduisions point de larges extraits.

Voicy vostre Nouvelle France aux pieds de V. M. Une troupe de Barbares,... l'a reduitte aux abois. Ecoutez, SIRE, si vous l'avez pour agréable, sa voix languissante, \& ses dernieres paroles: Sauvez-moy, s'écrie-t-elle, je vay perdre la Religion Catholique: on me va ravir les Fleurs de Lys: je ne seray plus Françoise, on me dérobe ce beau Nom, dont j'ay esté honorée depuis si long-temps: je tomberay antre les mains des étrangers, quand les Iroquois auront tiré le reste de mon sang, qui ne coule quasi plus: je seray bien-tost consommée dans leurs feux: \& le Demon va enlever un grand nombre de Nations, qui attendroient le salut de vostre Pieté, de vostre Puissance, \& de vostre Generosité. SIRE, voila les soupirs \& les sanglots de cette pauvre affligée...

$21 \mathrm{Si}$ nous tenons compte des évaluations faites par Renaud, 296 personnes seraient passés en Nouvelle-France dans les années 1608-1640, et 964 entre 1640 et 1660 . Ibid., 43. Or Langlois estime à 500 personnes le nombre d'émigrés au pays entre 1608 et 1653; ce qui donnerait pour la période $1640-1653,204$ personnes $(500-296)$; et $760(964-204)$ pour la période 1654-1660. Voilà pourquoi nous prêtons plus particulièrement attention à cette seconde période.

${ }^{22}$ L. Pouliot, Etude sur les Relations des Jésuites, 285-286.

23 E. Salone, La Colonisation de la Nouvelle-France, 102. Le P. Pouliot abonde dans le même sens que Salone. «Le but que se proposaient les Relations, écrit-il, ... c'était surtout de créer en France ce qu'on appelle aujourd'hui un mouvement d'opinion contre les Iroquois. Et il serait téméraire d'affirmer qu'elles n'ont pas largement préparé l'envoi des troupes. » L. Pouliot, Etude sur les Relations des Jésuites, 264.

24 E. Salone, La Colonisation de la Nouvelle-France, 102. 
Si vous consultez le Ciel, il vous dira que vostre salut est peut-estre enfermé dans le salut de tant de Peuples de V. M. Si vous considerez le nom François, vous sçaurez, SIRE, que vous estes un grana Roy, qui faisant trembler l'Europe, ne doit pas estre mesprisé dans l'Amerique. Si vous regardez le bien de vostre Estat: vostre esprit, qui voit à l'âge de vingt-quatre ans, ce que plysieurs grands Princes ne voyent pas à cinquante, connoistra combien la perte d'un si grand paîs sera dommageable à vôtre Royaume.

Ce coup [rendre la santé à la Nouvelle France] est reservée à V. M. qui sauvant les corps \& les biens de sa Colonie Françoise, \& les ames d'un tres-grand nombre de Nations, les obligera toutes de prier Dieu, qu'il vous fasse porter le nom de Saint, aussi bien qu'à vostre grand Ayeul, dont vous imiterez le zele, entreprenant une guerre sainte. ${ }^{25}$

Ce que nous avons dit de l'activité du P. Le Jeune jusqu'à maintenant, aura suffi, nous l'espérons, à nous convaincre de la part importante qu'il aura prise dans l'histoire de la colonisation en Nouvelle-France dans la première moitié du $\mathrm{XVII}^{\mathrm{e}}$ siècle. Si on a prétendu, et avec raison, que la restitution de la colonie en 1632 «eut été vaine si les initiatives particulières et, au premier rang, les initiatives religieuses n'étaient intervenues $\gg,{ }^{26}$ et qu' « en cette genèse », la Compagnie de Jésus a eu à jouer un « rôle de tout premier plan », serait-il exagéré d'affirmer que le $P$. Le Jeune apparaît comme une des figures les plus représentatives de l'effort colonial à l'époque. Quoi qu'il en soit, le P. Le Jeune est vraiment le «fondateur de la Mission canadienne » 27 et, avec Champlain, la préfiguration de ce que seront un jour ces grandes figures d'intendants, les Talon, les De Meulles et les Dupuy.

\section{Gilles Chaussé, s.j.*}

$25 \mathrm{JR}, 46: 196-198$.

26 G. Rigault et G. Goyau, Les Martyrs de la Nouvelle-France, 14.

27 Cette assertion est du docteur E. B. O'Callaghan. C. de Rochemonteix, Les Jésuites et la Nouvelle-France au XVIIe siècle, 1: 191.

* On voudra bien retenir le véritable prénom de notre collaborateur: Gilles. C'est par erreur que, à la fin de la première tranche de son article (livraison de juin de la Revue, nous lui avons prêté, celui de: Jules. (NDLD). 